\title{
Influence of Picric Acid on the SHG Efficiency of L-Prolinium Tartrate Crystals
}

\section{S. Natarajan ${ }^{1 *}$, K. Moovendaran ${ }^{1}$, J. Kalyana Sundar ${ }^{1}$, G. Bhagavannarayana ${ }^{2}$ and S.A. Martin Britto Dhas ${ }^{1}$}

\author{
${ }^{1}$ School of Physics, Madurai Kamaraj University, Madurai-625 021, India \\ ${ }^{2}$ Material Characterization Division, National Physical Laboratory, New Delhi 110012, India \\ * Corresponding author: s_natarajan50@yahoo.com
}

\begin{abstract}
Single crystals of the NLO material L-Prolinium tartrate (LPT) have been grown in the presence and absence of picric acid, by slow evaporation technique. Good quality crystals were harvested within 30 days. The grown crystals were characterized by single crystal X-ray diffraction. The quality of the crystals grown in the presence and absence of picric acid was examined by high resolution X-ray diffractometry (HRXRD). Fourier transform infrared spectroscopy (FTIR) and Fourier transform Raman spectroscopy were used to confirm the presence of the functional groups of L-Prolinium tartrate and the absence of picric acid in the crystals. The thermal stability and the trend of decomposition of the grown crystals were analyzed by thermo gravimetric analysis (TGA). The influence of picric acid on the second harmonic generation efficiency was studied by Kurtz and Perry method. It was observed that the SHG efficiency increased by about 2.3 times compared to that of normal LPT crystals. The results are discussed in detail.
\end{abstract}

Keywords: organic compounds; crystal growth; X-ray diffraction; thermo-gravimetric analysis

\section{INTRODUCTION}

In recent years, several efforts have been made to develop new inorganic, organic and semiorganic nonlinear optical (NLO) crystals [1-5]. The science and technology of crystal growth has advanced rapidly for the development of new NLO materials for several applications in telecommunication, optical data storage, optical information processing, optical switching, frequency conversion and electro-optical modulation [6]. Second harmonic generation (SHG) or frequency doubling is the most readily understood and widely used property of the NLO 
phenomenon, which allows for example the production of high intensity green laser pointers [7]. One way to improve the above properties is growing the crystal in different chemical environments. The presence of additives or impurities during the crystallization is one of the most important factors that affect the morphology and the physical properties of the crystal. Podder [8] reported that the presence of $\mathrm{KCl}$ in the growth medium suppresses the metal-ion impurities and increases the growth rate. The increase in the quality of potassium dihydrogen phosphate (KDP) crystals in the presence of $\mathrm{KCl}$ is due to the complexation of trace metalion impurities in solution by $\mathrm{Cl}^{-}$ions. Hence, it was planned to investigate the influence of dopant in L-Prolinium tartrate (LPT) crystal, a SHG material. The structure of LPT was elucidated by Subha Nandhini et al., in 2001 [9] and the nonlinear optical and other characterizations were carried out in 2006 [10] from our laboratory. The second harmonic generation (SHG) efficiency of LPT crystal was found to be 1.2 times higher than that of KDP. In this paper, the crystalline perfection and SHG efficiency of LPT crystals in the presence and absence of picric acid are reported. The grown crystals were characterized using single crystal X-ray diffraction. The crystalline perfection was tested by high resolution Xray diffractometer. FTIR and FT-Raman studies were carried out to confirm the absence of picric acid inside the lattice. TGA/DTA studies and SHG studies were also carried out and the results are discussed.

\section{EXPERIMENTAL}

\subsection{Crystal Growth}

Single crystals of LPT $\left[\left(\mathrm{C}_{5} \mathrm{H}_{10} \mathrm{NO}_{2}\right)^{+}\left(\mathrm{C}_{4} \mathrm{H}_{5} \mathrm{O}_{6}\right)^{-}\right]$were grown by mixing L-Proline and L-Tartaric acid in 1:1 stoichiometric ratio in aqueous solution, using slow evaporation method. Optically clear and well-shaped crystals were obtained in a period of 15-20 days. These crystals were powdered and recrystallized in the presence of picric acid (about 1 mole \%). Good quality crystals (referred hereafter as PLPT,) were harvested in a period of 25-30 days.

\subsection{Characterization}

The grown crystals were subjected to single crystal X-ray diffraction using Nonius CAD4/MACH 3 diffractometer, with $\mathrm{MoK \alpha}$ radiation $(\lambda=0.71073 \AA$ ). The cell parameters were obtained from the least-squares refinement of the setting angles of 25 reflections. The FTIR spectra of the samples were recorded in the frequency region of $400-4000 \mathrm{~cm}^{-1}$ using a Bruker Alpha-p FTIR Spectrometer, at a resolution of $4 \mathrm{~cm}^{-1}$. Raman spectral measurements were made with a model RFS 100/S Bruker. Nd: YAG laser operated at $1064 \mathrm{~nm}$ with a power output of $200 \mathrm{Mw}$ used as source. The spectrum was recorded over the range 4000$500 \mathrm{~cm}^{-1}$. The sample was finely powdered and pressed into a small depression on a metal disc and mounted on the sample compartment. This instrument has a resolution of $4 \mathrm{~cm}^{-1}$. The second harmonic generation (SHG) conversion efficiency was tested using a modified setup of Kurtz and Perry. The fundamental beam of $1064 \mathrm{~nm}$ from a Q switched Nd:YAG laser was used to test the SHG efficiency of LPT and PLPT crystals. The laser beam used had 
the pulse energy $3.5 \mathrm{~mJ} /$ pulse and pulse width $8 \mathrm{~ns}$ and repetition rate $10 \mathrm{~Hz}$. A photo multiplier tube (Hamahatsu R2059) was used as detector and 90 degree geometry was employed. The input laser beam was directed on the microcrystalline powdered sample packed in a capillary tube of diameter $0.154 \mathrm{~mm}$. The assembly of an oscilloscope and photomultiplier detector was employed to measure the emitted light by the sample. The SHG signal generated in the sample was confirmed from the emission of green radiation from the sample. The optical signal generated from the sample was converted into an electrical signal and measured on the oscilloscope. Simultaneous thermogravimetric analysis (TGA) and differential thermal analysis (DTA) were carried out for the crystals, using a SDT Q600 V8.3 build 101 thermal analyzer. A powder sample was used for the analysis in the temperature range of $26^{\circ} \mathrm{C}$ to $800^{\circ} \mathrm{C}$ with a heating rate of $20^{\circ} \mathrm{C} / \mathrm{min}$. The crucible used was of alumina $\left(\mathrm{Al}_{2} \mathrm{O}_{3}\right)$, which also served as a reference for the sample.

\section{RESULTS AND DISCUSSION}

\subsection{Single Crystal X-ray Diffraction}

The unit cell dimensions obtained are listed in Table 1. It is seen that the crystal belongs to the monoclinic system with the space group $\mathrm{P} 2{ }_{1}$. The cell dimensions agree well with the reported values [9]. The density of pure and PLPT crystals was found to be $1.55(1) \mathrm{g} / \mathrm{cm}^{3}$ and it is in good agreement with the literature values $\left(1.55 \mathrm{~g} / \mathrm{cm}^{3}\right)$. No appreciable changes in the values of the cell parameters indicate that the inclusion of picric acid in the lattice of LPT is not to an appreciable extent.

Table 1: XRD data for LPT and PLPT

\begin{tabular}{|l|l|c|l|}
\hline \multicolumn{1}{|c|}{$\begin{array}{c}\text { Lattice } \\
\text { Parameters }\end{array}$} & $\begin{array}{c}\text { Literature } \\
\text { value }\end{array}$ & LPT & \multicolumn{1}{c|}{ PLPT } \\
\hline $\mathrm{a}(\AA)$ & $5.007(1)$ & $5.009(2)$ & $5.003(2)$ \\
$\mathrm{b}(\AA)$ & $17.673(3)$ & $17.678(2)$ & $17.684(9)$ \\
$\mathrm{c}(\AA)$ & $6.523(1)$ & $6.514(2)$ & $6.523(3)$ \\
$\beta\left({ }^{\circ}\right)$ & $100.40(2)$ & $100.54(3)$ & $100.36(3)$ \\
$\mathrm{V}\left(\AA^{3}\right)$ & $567.8(2)$ & $567.1(7)$ & $567.6(4)$ \\
\hline
\end{tabular}

\subsection{Multi-Crystal X-ray Diffraction}

The crystalline perfection of the grown single crystals was characterized by HRXRD by employing a multicrystal X-ray diffractometer developed at NPL [11]. The well-collimated and monochromated $\mathrm{MoK} \alpha_{1}$ beam obtained from the three monochromator Si crystals set in dispersive $(+,-,-)$ configuration has been used as the exploring X-ray beam. The specimen crystal is aligned in the $(+,-,-,+)$ configuration. Due to dispersive configuration, though the lattice constant of the monochromator crystal(s) and the specimen are different, the unwanted 
dispersion broadening in the diffraction curve (DC) of the specimen crystal is insignificant. The specimen can be rotated about the vertical axis, which is perpendicular to the plane of diffraction, with minimum angular interval of 0.4 arc sec. The rocking or diffraction curves were recorded by changing the glancing angle (angle between the incident X-ray beam and the surface of the specimen) around the Bragg diffraction peak position $\theta_{\mathrm{B}}$ (taken as zero for the sake of convenience) starting from a suitable arbitrary glancing angle and ending at a glancing angle after the peak so that all the meaningful scattered intensities on both sides of the peak include in the diffraction curve. The DC was recorded by the so-called $\omega$ scan wherein the detector was kept at the same angular position $2 \theta_{\mathrm{B}}$ with wide opening for its slit.

Before recording the diffraction curve to remove the non-crystallized solute atoms which remained on the surface of the crystal and also to ensure the surface planarity, the specimen was first lapped and chemically etched in a non preferential etchant of water and acetone mixture in 1:2 volume ratio.

Fig. 1(a) and (b) shows the high-resolution diffraction curves (DCs) recorded respectively for LPT and PLPT single crystal specimens grown by slow evaporation solution growth technique (SEST) using (040) diffracting planes in symmetrical Bragg geometry by employing the multicrystal X-ray diffractometer with $M o K \alpha_{1}$ radiation. Form the figure 1(a), it is clear that the DC of the undoped specimen is not having a single peak but contains four well resolved peaks. These additional peaks depict internal structural low angle (tilt angle > 1 arc min but less than a deg.) boundaries [12] whose tilt angles (the mis-orientation angle between the two crystalline regions on both sides of the structural grain boundary) are 302 , 168 and 326 arc s from their adjoining regions. The full width at half maximum (FWHM) of these peaks are respectively 105, 150, 60 and 184 arc s.

As seen in curve (b) the doped specimen contains only one clear low angle boundary with tilt angle 356 arc s. It is worth to observe the prolonged scattered intensity at higher glancing angles from the peak position of the low angle boundary. This type of scattered intensity at higher diffraction angles shows that the crystal contains interstitial type of point defects and their clusters probably due to dopants (picric acid) which may be explained in the following way. Due to interstitial defects (self interstitials or impurities or dopants at interstitial sites), the lattice around these defects undergo compressive stress [13] and the lattice parameter $d$ (interplanar spacing) decreases and leads to give more scattered (also known as diffuse X-ray scattering) intensity at slightly higher Bragg angles $\left(\theta_{B}\right)$ as $d$ and $\sin \theta_{B}$ are inversely proportional to each other in the Bragg equation $\left(2 d \sin \theta_{B}=n \lambda ; n\right.$ and $\lambda$ being the order of reflection and wavelength, respectively which are fixed). The inset in curve (b) shows the schematic to illustrate how the lattice around the defect core undergoes compressive stress. The converse explanation is true in the case of vacancy defects which cause tensile stress in the lattice around the defect core leading to increase of lattice spacing which in turn results in more scattered intensity at the lower Bragg angles. It is worth to mention here that the observed scattering due to point defects is of short range order as the strain due to such minute defects is limited to the very defect core and the long range order could not be 
expected and hence the change in the lattice parameter of the overall crystal may not be possible. It may be mentioned here that the minute information like the asymmetry in the DC could be possible as in the present sample only because of the high-resolution of the multicrystal X-ray diffractometer used in the present investigation.

From the above discussion and comparison of curves (a) and (b), it is evident that the asgrown crystal of LPT has a tendency to grow with grain boundaries and when grown with picric acid (as dopant), it seems the crystalline perfection improved to a significant extent. However, we cannot reduce completely the formation of low angle boundaries.
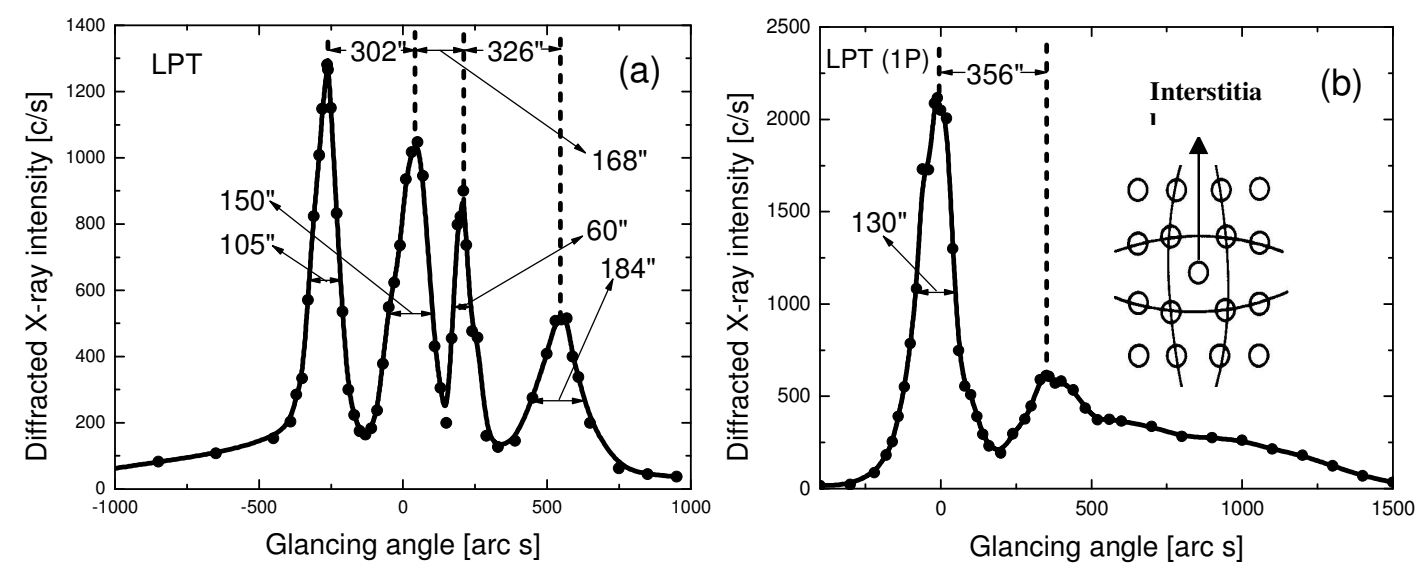

Fig. 1. High-resolution X-ray diffraction curves recorded for (040) diffracting planes in symmetrical Bragg geometry using $\mathrm{MoK} \alpha_{1}$ radiation for LPT single crystals: (a) LPT and (b) PLPT

\subsection{TGA/DTA Analysis}

Thermo gravimetric analysis has been carried out to investigate the changes in the thermal behavior of the LPT and PLPT. The crystals of PLPT also showed the same trend of decomposition as that of LPT. The interpretation of the thermal analysis has already been reported earlier [10].

\subsection{Fourier Transform Infra-red and Fourier Transform Raman Spectroscopic Analysis}

The FTIR and FT-Raman spectroscopic studies were carried out to analyze qualitatively the presence of functional groups in the LPT and PLPT crystals. The spectra of LPT and PLPT are shown in Fig. 3 and Fig. 4 The interpretation of FTIR is described in our earlier report [10]. Fig. 3 and Fig. 4 show that the FTIR spectra of LPT and PLPT are identical as is the case with their FT-Raman spectra. 


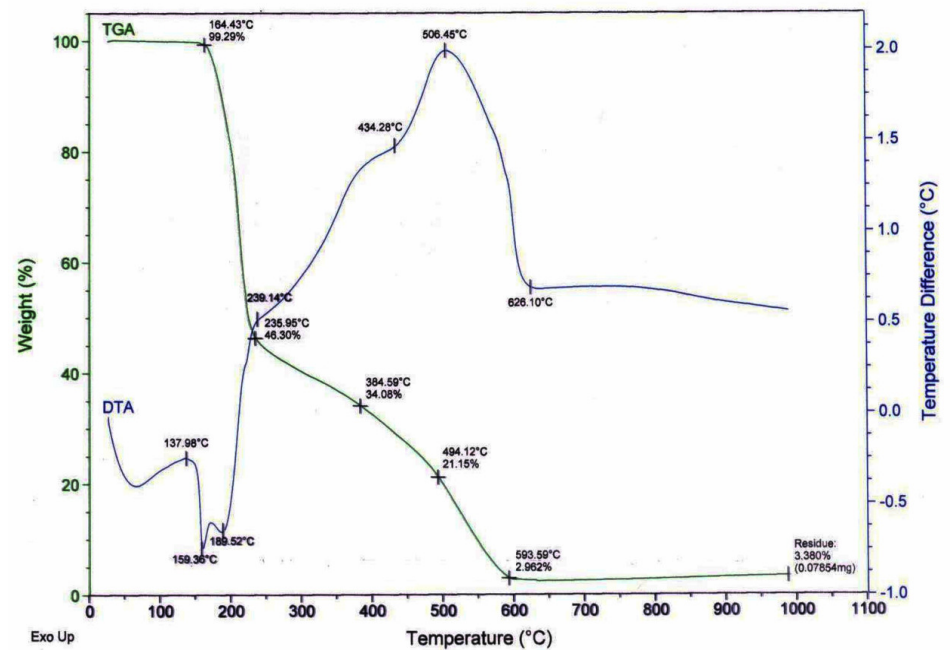

Fig 2. TGA/DTA of PLPT crystal

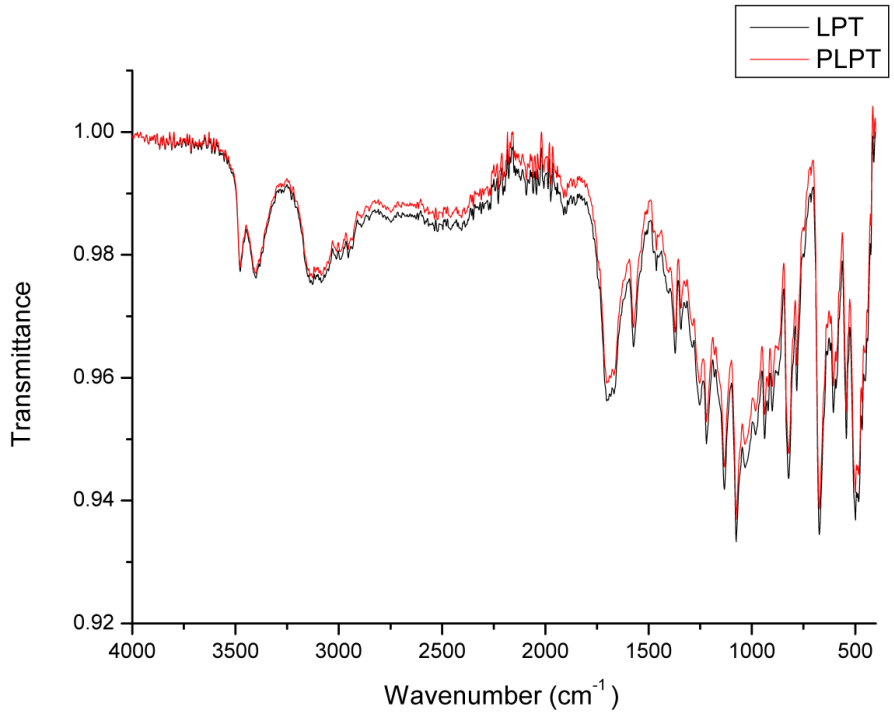

Fig. 3 FTIR spectra of LPT and PLPT 


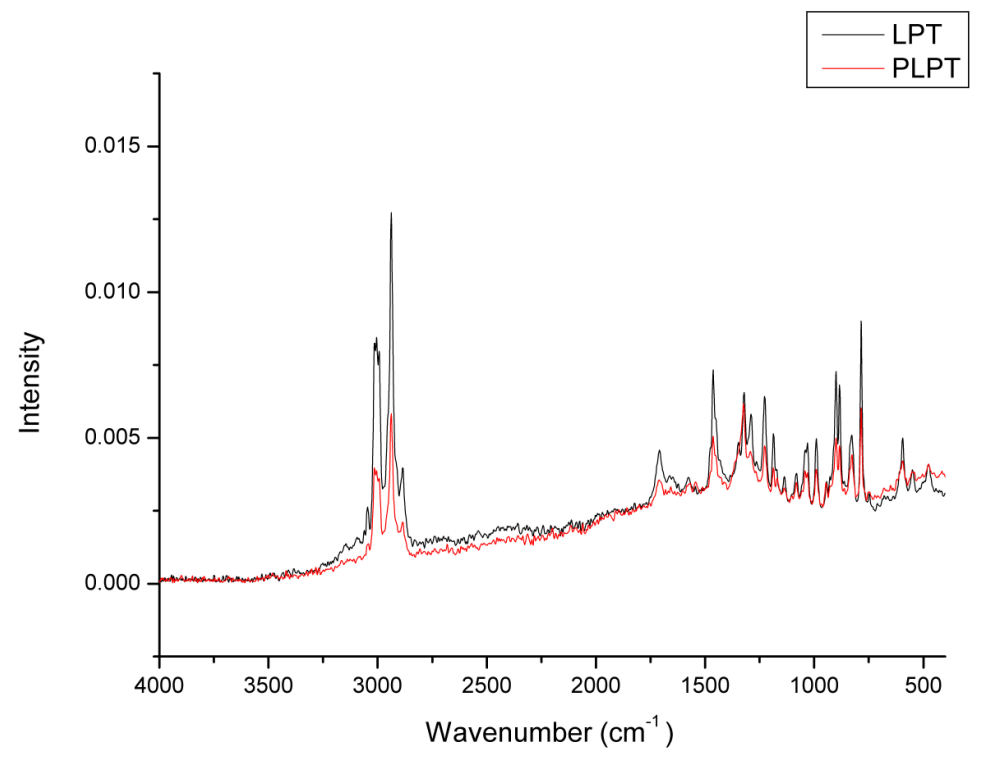

Fig. 4 FT-Raman spectra of LPT and PLPT

The Raman bands of the compound around $3010 \mathrm{~cm}^{-1}$ are attributed to $\mathrm{O}-\mathrm{H}$ stretching vibration of the carboxyl group. The very strong peak at $2935 \mathrm{~cm}^{-1}$ and the shoulder peak at $2883 \mathrm{~cm}^{-1}$ are due to $\mathrm{CH}_{2}$ stretching. The medium peak at $1712 \mathrm{~cm}^{-1}$ is assigned to the stretching vibration of $\mathrm{C}=\mathrm{O}$ group. The weak peak at $1560 \mathrm{~cm}^{-1}$ is attributed to the in-plane bending of the amino group. The medium intense peaks at 1321, 1227 (wagging), 1188 (twisting), $828 \mathrm{~cm}^{-1}$ and the strong peak $784 \mathrm{~cm}^{-1}$ (rocking) are assigned for the different modes of vibrations of $\mathrm{CH}_{2}$. The weak peaks at $993 \mathrm{~cm}^{-1}$ and $902 \mathrm{~cm}^{-1}$ are assigned to proline ring stretching. The strong peak at $884 \mathrm{~cm}^{-1}$ is assigned to the combination of ring stretching and $\mathrm{CH}_{2}$ rocking. The peaks at 553 and $472 \mathrm{~cm}^{-1}$ are due to the skeletal deformation and COO rocking, respectively. No peaks are observed due to the functional groups such as nitro group or aromatic vibrations; which reveal the absence of picric acid molecules in the crystals.

\subsection{SHG Measurement}

The preliminary testing of materials for second harmonic generation was carried out by using a modified setup of Kurtz and Perry [14]. The SHG conversion efficiency of PLPT was found to have enhanced compared to that of LPT. The measured output for pure LPT and PLPT were $15 \mathrm{mv}$ and $34 \mathrm{mv}$, respectively. This shows that the SHG conversion efficiency of PLPT is 2.3 times greater than that of LPT. The enhancement in SHG efficiency of PLPT may be due to the crystalline perfection. 


\section{CONCLUSIONS}

Single crystals of L-Prolinium tartrate, a new organic nonlinear optical crystal have been grown in the presence and absence of picric acid by slow evaporation technique. The grown crystals were characterized by single crystal X-ray diffraction. The HRXRD reveals that the as-grown crystal of LPT has a tendency to grow with grain boundaries and when dopants (picric acid) are added, it seems that the crystalline perfection improved to a significant level. The crystals of PLPT also showed the same trend of thermal decomposition as that of LPT. Fourier transform infrared and FT-Raman spectroscopic studies showed the absence of picric acid in the crystals of L-Prolinium tartrate. Also, the vibrational modes of the functional groups of both the crystals have been identified in the Raman spectra. The Kurtz and Perry method revealed that the SHG efficiency increased significantly. It may be due to the crystalline perfection.

\section{ACKNOWLEDGEMENT}

One of the authors (SN) thanks the CSIR for the funding provided under the Emeritus Scientist Scheme.

\section{REFERENCES}

[1] Chenthamarai, S., Jayaraman, D., Subramanian, C., and Ramasamy, P., (2001), "Mechanical and optical studies on pure and nitro doped 4-hydroxyacetophenone" Materials letters, 47, $247-251$

[2] Rajesh, N.P., Kannan, V., Santhana Raghavan, P., Ramasamy, P., and Lan,C.W., (2002) "Nucleation studies and crystal growth of $\left(\mathrm{NH}_{4}\right) \mathrm{H}_{2} \mathrm{PO}_{4}$ doped with thiourea in supersaturated aqueous solutions" Materials Chemistry and Physics, 52, 326 -328

[3] Hu, Z.G., Yoshimura, M., Mori, Y., Sasaki, T., (2005) "Design and growth of new NLO crystals for UV light generation” J. Crystal Growth, 275, 232 -239

[4] Ambujam, K., Rajarajan, K., Selvakumar, S., Joseph, A., and P. Sagayaraj, P., (2006) "Growth and characterization of a novel NLO crystal bis-glycine hydrogen chloride (BGHC)" J. Crystal Growth, 286 (2006) 440-444

[5] Hussaini, S.S., Dhumane,N.R., Rabbani, G., Karmuse, P., Dongre,V.G., and Shirsat, M.D., (2007) "Growth and high frequency dielectric study of pure and thiourea doped KDP crystals" Crystal Research and Technology, 42,1110 -1116

[6] Arivuoli, D., (2001) “J. Phys. 57, 871

[7] Coe, B.J., (2006) "Switchable Nonlinear Optical Metallochromophores with Pyridinium Electron Acceptor Groups" Accounts of Chemical Research, 39 (2006), 383-393

[8] Podder, J., Ramalingom, S., and Narayana kalkura, S., (2001) "An Investigation on the lattice distortion in Urea and $\mathrm{KCl}$ Doped KDP single crystals by X-ray Diffraction studies "Crystal Research and technology, 36, 549-556.

[9] Subha Nandhini, M., Krishnakumar, R.V., and Natarajan, S., (2001) "L-Prolinium tartrate", Acta Crystallograpica, C57, 423-424 
[10] Martin Britto Dhas, S.A., and Natarajan, S., (2007) "Growth and characterization of Lprolinium tartrate - A new organic NLO material” Crystal Research and technology, 42, 471-476

[11] Lal, K., and Bhagavannarayana, G., (1989) "A high-resolution diffuse X-ray scattering study of defects in dislocation-free silicon crystals grown by the float-zone method and comparison with Czochralski-grown crystals" J. Applied Crystallography, 22, 209-215

[12] Bhagavannarayana, G., Ananthamurthy, R.V., Budakoti, G.C., Kumar, B., and Bartwal, K.S., (2005) "A study of the effect of annealing on Fe-doped LiNbO3 by HRXRD, XRT and FT-IR" J. Applied Crystallography, 38, 768-771

[13] Bhagavannarayana, G., Parthiban, S., and Meenakshisundaram, S., (2008) "An Interesting Correlation between Crystalline Perfection and Second Harmonic Generation Efficiency on KCl- and Oxalic Acid-Doped ADP Crystals" Crystal Growth and Design, $8,446-451$

[14] Kurtz, S.K., and Perry, T.T., (1968) "A Powder technique for the evaluation of nonlinear optical materials” J. Applied Physics, 39, 3798 -3812 